\title{
Linx
}

Revue des linguistes de l'université Paris X Nanterre

$7 \mid 1995$

Saussure aujourd'hui

\section{Signe et temporalité dans les notes item}

\section{Francis-Marie Gandon}

\section{(2) OpenEdition}

\section{Journals}

Édition électronique

URL : http://journals.openedition.org/linx/1134

DOI : 10.4000/linx.1134

ISSN : 2118-9692

\section{Éditeur}

Presses universitaires de Paris Nanterre

\section{Édition imprimée}

Date de publication : 1 septembre 1995

Pagination : 147-151

ISSN : 0246-8743

\section{Référence électronique}

Francis-Marie Gandon, «Signe et temporalité dans les notes item », Linx [En ligne], 7| 1995, mis en ligne le 12 juillet 2012, consulté le 10 décembre 2020. URL : http://journals.openedition.org/linx/1134 ; DOI : https://doi.org/10.4000/linx.1134

Ce document a été généré automatiquement le 10 décembre 2020.

Département de Sciences du langage, Université Paris Ouest 


\title{
Signe et temporalité dans les notes item
}

\author{
Francis-Marie Gandon
}

1 L'originalité flagrante des Notes item ${ }^{1}$ nous semble tenir dans une conception de la diachronie (un certain type de diachronie). comme opération mentale.

2 L'attitude saussurienne concernant le temps est faite de détours et de rétractations. L'opposition cardinale diachronie/synchronie a cependant tout lieu d'être considérée non seulement comme la vulgate de l'oeuvre mais encore comme l'essentiel d'un projet avant tout dichotomique. Trois points résumeront cette « vulgate essentielle ».

1. L'évolution historique n'explique en rien tel état synchronique de langue. Coups et positions d'échecs se sont superbement inutiles.

2. L'évolution historique n'aide en rien à la compréhension du signe dont elle rend par ailleurs l'identité problématique : leitmotiv des Notes sur la légende germanique ${ }^{2}$.

3. Les mêmes facteurs servent de loi à la crise et à l'état (métaphore politique).

3 Fixées dès 1894 , dans des notes préparatoires à une conférence sur Whitney ${ }^{3}$ ces points de vue remontent à une réflexion largement antérieure selon Saussure lui-même.

4 Dans le «fragment sémiologique central » que constituent selon Christian Stetter ${ }^{4}$ les Notes item. un autre type de diachronie apparaît, indirect et discret - si discret qu'il faut le détecter, à travers quelques rares métaphores et expressions, à grands efforts. Ce type de diachronie concerne le signe - ici appelé « sème (re)colligible ».

5 Commençons par le premier type d'indice - à peu près unique : l'image de la lanterne magique.

«Item. De la psychologisation des signes vocaux. -

A. Supposons que sur le même disque de lanterne magique on donne successivement

Disque vert

disque jaune

disque noir

disque bleu

disque bleu (de nouveau) 


\begin{abstract}
disque rouge
disque violet

[en marge : Après cela :Ortoute la particularité du mot, est d'être un sème colligible, mais reposant sur la succession des syllabes.]

Il résulterait de l'ensemble de ces signes, la quasi-impossibilité pour la mémoire de se les représenter dans leur suite, ou «comme une suite recolligible [ces quatre derniers mots soulignés, la soulignure ensuite biffée] faisant un tout. »

B. Supposons en second lieu qu'on ne fasse pas <se> succéder [en marge : Mettre ici carrément /vert/jaune/noir/] ces couleurs ${ }^{5}$, mais mais (sic) qu'on les juxtapose sur le disque (à gauche vert, ensuite jaune, etc... jusqu'à la droite). On aura dans ce cas une figure sinon recolligible à tout le monde, du moins commençant à devenir (re) [barré] colligible et à être une figure.

C. Il a donc fallu pour que la figure visuelle devînt figure abandonner le principe de la succession temporelle, et recourir à [signe inintelligible] (N. It., fo 12 ).
\end{abstract}

De la sorte le signe s'apparente à une opération mentale de type synthétique basée sur une portion aussi petite que l'on voudra de diachronie. Cette opération mentale inconsciente produit la conscience d'une unité sémiologique. Sémiologie tout-à-fait particulière puisque fondée sur une diachronie (métaphore du cercle) dont l'expression consciente est la linéarité spatiale : «principe de l'unispatialité » ( $\left.f^{\circ} 13\right)$. Elle s'oppose alors radicalement à un objet sémiologique non unispatial: idéogrammes tableau allégorique, peinture quelconque (exemples donnés par Saussure). Ce type de sème est en effet spatial a priori alors qu'il faut insister sur le point fondamental suivant : la divisibilité du sème unispatial n'est qu'un après-coup et s'apparente à la dissection d'un cadavre (métaphore surdéterminée par la paronomase sème/sôme - ce dernier terme substitué à « signifiant »), ou encore la transformation d'un organisme en mécanisme, d'une physiologie en anatomie. On ne comprendrait autrement d'aucune façon l'adversatif dans l'expression déjà citée de "sèmecolligible mais reposant sur la succession des voyelles" (nous soulignons).

Mais dans cette question il faut donner grande attention au "mérisme " (à la divisibilité dans le temps) des parties du mots (sic) ; c'est cette divisibilité [qui](barré) de la chaîne sonore qui peut-être plus que la variété des sons contribue à imposer l'illusion de groupes organiques. Il est merveilleux en somme de pouvoir mettre des tirets comme $\lambda v-\Theta \eta-\sigma o ́-\mu \varepsilon v o-s . »\left(f^{\circ} 16, v e r s o\right)$.

7 Ce second type d'indice (expression linguistique) éclaire l'acharnement mis par Saussure à dénier toute pertinence sémiologique à une quelconque morphologie. En tant qu' « unité vivant en dehors de tout discours dans le trésor mental » ( $\left.f^{\circ} 22\right)$, en tant qu' « il se dégage sans analyse » (ibid.), le mot - l'unité du mot - récuse tout découpage spatial ou, à tout le moins, assimile ce dernier à une manière d'illusionnisme, de fantasmagorie ( "merveilleux »). On imagine - s'il fallait prendre au mot le maitre de Genève - à quelles insurmontables difficultés la linguistique serait confrontée, pour quelque chose d'aussi banal par exemple que la désinence verbale ou la déclinaison!

8 L'important est qu'ici soit récusée une correspondance terme à terme entre éléments du signifiant et du signifié (Saussure préfigure donc partiellement en ceci l'isomorphisme) et donc reniée toute linguistique à venir fondée sur des distinguos entre morphèmes. Dans ce cadre théorique un problème annexe prend tout son sens : celui de la «faculté de notre esprit de s'attacher à un terme en soit nul » (f॰ 12) - donc de donner au degré zéro la définition qu'en proposera plus tard Barthes d'«absence qui signifie », de même que la sortie assez hargneuse à l'encontre de Bréal et plus précisément de sa "fameuse ellipse » (f॰ 1 verso). C'est qu'en effet il n'y a nulle raison de recourir à une parité entre éléments de l'« aposème » et de la «signification » (pour recourir à une terminologie en vigueur 
dans les Notes item). Nous sommes donc ici aux antipodes de la feuille de papier, avec une expression non proportionnés à un contenu non limitable priori.

Résumons : a) la linéarité du signifiant implique une diachronie actualisante - en aucun cas un temps analytique. Fondée sur cette linéarité la langue se distingue de façon cardinale de tout autre objet sémiologique (de nature achronique et spatiale).

b) l'unité linguistique est le produit d'une opération mentale : la (re)colligibilité du sème de nature inconsciente, synthétique, micro-diachronique. Une analyse consciente, spatiale, aposteriorique du mot - comme l'est la morphologie - n'est qu'un après coup : une commodité de représentation.

11 Il en résulte que les Notes item proposent une réponse à la question considérée ailleurs notamment dans l'analyse de la légende - comme insoluble, celle de l'identité , et esquissent une théorie de la faculté de compréhension ("psychologisation») de cette identité. En ce sens une inférence théorique supplémentaire est nécessaire : la diachronie " opérative » doit, d'une certaine façon, condenser la diachronie historique du mot - ce qui donne au terme de (re)colligibilité toute sa portée. Proposition on s'en doute indémontrable ( et peut-être au delà de toute "science ») elle n'en est pas moins à l'horizon de la réflexion saussurienne sur le temps.

Mais aussi bien à celui de la réflexion sur le signe. Ce dernier est décrit comme étant à l'intersection de la voie « historique » et de la voie « consciente » $\left(\mathrm{f}^{\circ} 20\right)$ - de la diachronie longues destructrice et de la diachronie brève appropriatrice. Mais cette appropriation procède aussi avec l'aide de la Proportion - qui seule permet par exemple de retrouver « noeud » dans « noueux » (sur le modèle langueur : langoureux). La Proportion est donc aussi donnée comme la condition du sens : c'est une proposition - absolument récurrente dans le Cours - sur quoi les Notes ltem raffinent d'ailleurs en adjoignant aux relations dissimile (valeur purement oppositive du signe en système) les relations simile (motivation relative du "parasème » au sein d'un paradigme sémantique). En d'autres termes plus elles s'approfondissent plus les notions de signification (sémiose) et de valeur divorcent ${ }^{7}$. Le signe n'est plus seulement le lieu d'une Aufhebung mais celui d'un écartèlement. C'est le prix à payer pour une théorie de plus en plus abstraite des identités mais aussi - et le point est fondamental - pour l'esquisse d'une théorie de la conscience de ces identités, c'est-à-dire de la faculté de langage ${ }^{8}$.

\section{NOTES}

1. Comportant une vingtaine de feuillets ces notes sont rassemblées à la Bibliothèque publique et universitaire de Genève salle Sénébier. Elles ont été publiées pour la première fois par R. Engler dans son édition critique du Cours de linguistique générale : t. II Wiesbaden, 1974.

2. Notes relatives à un cours professé en 1904. Essentiellement consacrées au Nibelungenlied elles comportent dix-huit cahiers et deux enveloppes. Conservées salle Sénébier.

3. Ébauche d'un article consacré au linguiste américain, mort le 7 Juin 1894. Notes conservées salle Sénébier et publiées par R. Engler (1974). 
4. «La fonction des réflexions sémiologiques dans la fondation de la linguistique générale chez $\mathrm{F}$. de Saussure », Kodikas-Code, Gunter Narr Verlag, Tübingen, vol. 1, n 1, janvier 1979, p. 12.

5. Ou « contours ", selon la leçon de R. Engler (1974: 38).

6. L'impossibilité d'approcher ce qu'est l'unité ou l'identité du symbole est une constante de l'analyse de la légende germanique. Voir entre autres les images du «fantôme » et de la «bulle de savon ".

7. Point sur lequel insiste particulièrement C. Stetterg in «Die Idee der Semiologie bei F. de Saussure » [L'idée de sémiologie chez F. de Saussure], Deutsche Sprache, Heft 4, 1976.

8. Il est curieux que la sensibilité saussurienne au temps ait échappé à l'intuition d'un penseur comme G. Guillaume, même ne disposant que de la vulgate: «Un facteur dont la formule saussurienne ne tient pas compte (a), et dont en toute question linguistique il y a lieu de tenir le compte le plus étroit, c'est le facteur temps. Le langage comme tout, comme intégrale, enveloppe une successivité : celle du passage de la langue - présente dans le sujet parlant en permanence (en dehors de toute momentanéité par conséquent) - à la parole, présente en lui par momentanéités seulement (par momentanéités plus ou moins espacées) ». Leçon du 20 février 1948, série C. in Principes de linguistique théorique, Les Presses de l'Université Laval, Québec et Librairie Klincksieck, Paris, 1973, p. 68. (a) Il s'agit de l'équation : langage = langue + parole. 